\title{
Influence of Combination Pharmacotherapy on Proinflammatory Gene COX-2 Expression in Skin Cancer
}

\author{
M. Woźniak*, C. Paluszkiewicz and W. Kwiatek \\ Institute of Nuclear Physics, Polish Academy of Sciences, PL-31342, Krakow, Poland
}

\begin{abstract}
Skin cancers are frequently occurring neoplastic changes in the human body. Among them, one of the most serious and dangerous are melanomas, meaning malignant neoplastic forms, which are characterized by a high risk of metastasis to the other organs. Currently, surgical removal is the most effective method of melanoma treatment, but due to the complicated location of tumor, such as brain or eyeballs, unfortunately not all cases undergo surgery. Second option is chemotherapy, which is very often associated with many side effects. Therefore, modern and innovative pharmacotherapy is needed. Here, we proposed a combination strategy based on administration of low doses of BRAF kinase inhibitor with mGluR $_{1}$ antagonist in melanoma cell line. Detection of proinflammatory COX-2 gene expression in melanoma (SK-MEL-2) cell line was performed by the polymerase chain reaction (realtime PCR) technique. Our results showed that simultaneous administration of low doses of tested compounds significantly decreased COX-2 expression in SK-MEL-2. Conducted research may be a first step to effectively to reduce the side effects while maintaining the therapeutic effect in melanoma treatment.
\end{abstract}

DOI: 10.12693/APhysPolA.137.62

PACS/topics: melanoma, BRAF kinase inhibitor, mGluR 1 antagonist, real-time PCR

\section{Introduction}

Melanoma, a skin cancer, is one of the most malignant human neoplasm. The number of this tumor cases is increasing at an alarming rate $[1,2]$. Moreover, melanoma cells are characterized by exceptional resistance to currently used treatment such as radiotherapy $[3,4]$ or chemotherapy. Therefore, from the last decade the main goal of oncological research is better understanding of neoplasm mechanisms and synthesis of innovative anticancer drugs. One of them is dabrafenib, a BRAF kinase inhibitor, which suppresses the excessive cell proliferation through MAPK kinase pathway inhibition [5]. Although this drug is effective in melanoma treatment, it is associated with many serious side effects [6] such as hyperkeratosis, disorders of internal organs and even increasing risk of cancer morbidity in other location than skin. Moreover, hyperactivity of glutamate system plays an important role in cancerous process and contributes uncontrolled cell proliferation $[7,8]$. Therefore, in this research, we present a conception of combination of pharmacotherapy based on simultaneous administration of $\mathrm{mGluR}_{1}$ antagonist (JNJ16259685) with a BRAF kinase inhibitor (dabrafenib) at low concentrations in the malignant melanoma cell line. In our study we focused on cyclooxygenase 2 (COX-2) expression, which is a proinflammatory gene strongly related with neoplasm process [9].

*corresponding author; e-mail: monika.wozniak@ifj.edu.pl

\section{Material and methods}

\subsection{Cell line}

For the study a human malignant melanoma (SK-MEL-2) cells line was used which was obtained from ATCC, USA. Melanoma cells were cultured with EMEM medium supplemented with $10 \%$ fetal bovine serum (ATCC, USA) and $1 \%$ penicillin-streptomycinneomycin solution (Sigma-Aldrich, USA). The cells were stored under constant environmental conditions in a humidified chamber of $5 \% \mathrm{CO}_{2}$ and $37^{\circ} \mathrm{C}$.

\subsection{Drugs}

Dabrafenib, a BRAF kinase inhibitor was purchased from Selleckchem, USA. Second drug, JNJ16259685, is a selective $\mathrm{mGlu}_{1}$ receptor antagonist which was obtained from Tocris, UK. Both compounds were dissolved in DMSO to $1 \mathrm{mM}$ dose and then re-diluted in distilled water to the final concentrations of 25,500 , and $2000 \mathrm{nM}$. Incubation with tested drugs was $72 \mathrm{~h}$ and then tests were performed.

\subsection{Real-time $P C R$}

The level of cyclooxygenase 2 gene (COX-2) expression related with inflammatory process was measured by polymerase chain reaction (real-time PCR) after drugs administration. This method consists 3 stages, in sequence: RNA isolation by RNeasy Mini Kit (Qiagen, Netherlands), reverse transcription with nanoScript kit (PrimerDesign, UK), and final $\mathrm{PCR}$ reaction with Precision PLUS mastermix (PrimerDesign, UK) using 
QuantStudio 7 flex Real-time System (Thermofisher, USA). The sequences of primers were as follows: GAPDH forward 5'-CCATGGAGAAGGCTGGG-3', reverse 5'-CAAAGTTGTCATGGATGACC-3', COX-2 forward 5'-TGAGCATCTACGGTTTGCTG-3', reverse 5'-TGCTTGTCTGGAACAACTGC-3', Genomed (Poland). The relative expression was performed by $2^{-\Delta \Delta C T}$ Livak's method [10].

\subsection{Statistical analysis}

The obtained data was expressed as a MEAN \pm standard deviation. One-way ANOVA followed by the Newman-Keuls post hoc test were performed. Statistical significance was assessed using GraphPad Prism 5.0 software and $\mathrm{P}$ value $<0.05$ was considered to be statistically significant.

\section{Results}

\subsection{Effects of simultaneous administration of dabrafenib and JNJ16259685}

Single administration of dabrafenib did not have effect on its own in cyclooxygenase 2 expression at $25 \mathrm{nM}$ dose, but paradoxically increased its level at the higher doses $500 \mathrm{nM}$ and $2000 \mathrm{nM}$ (Fig. 1a). Simultaneous administration of tested drugs significantly reduced COX-2 level at the lowest dose, but increasing doses was related with tendency to increase expression of cyclooxygenase 2 (Fig. 1b).
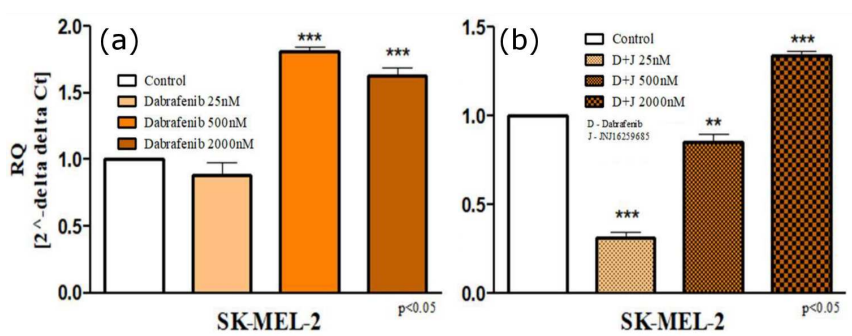

Fig. 1. Effects of single administration of dabrafenib (a) and simultaneous administration of dabrafenib and JNJ16259685 (b) on cyclooxygenase 2 expression in melanoma (SK-MEL-2) cells line.

\section{Summary}

Our results confirmed that using high doses of anticancer drugs is connected with toxicity and initiation of inflammatory process. Therefore, we postulated to decrease doses of compounds and administrate them together to obtain therapeutic effects while limiting side effects in the form of inflammation. Conducted results showed that targeted pharmacotherapy based on combination of dabrafenib and JNJ162685 in low doses may reverse inflammation, which is widely associated with cancerous process. Moreover, synergetic activity of tested drugs may allow for more effective inhibition of uncontrolled proliferation of cells by secondary messengers via cyclooxygenase 2 and finally it may contribute to degradation of melanoma tumor cells. Summarizing together, our research may be a significant step to develop modern and innovative drugs for melanoma treatment in the future.

\section{Acknowledgments}

This research was supported by scientific grant "Miniatura 2" which is carried out within the National Science Centre in Poland, project no. 2018/02/X/NZ3/00255. All experiments were performed using equipment purchased in the frame of the project cofunded by the Malopolska Regional Operational Program Measure 5.1 Krakow Metropolitan Area as an important hub of the European Research Area for 2007-2013, project No. MRPO.05.01.00-12-013/15.

\section{References}

[1] C. Garbe, K. Peris, A. Hauschild, P. Saiag, M. Middleton, L. Bastholt, J.J. Grob, J. Malvehy, J. NewtonBishop, A.J. Stratigos, H. Pehamberger, A.M. Eggermont, Eur. J. Cancer 63, 201 (2016).

[2] R. Siegel, K.D. Miller, A. Jemal, CA Cancer J. Clin. 62, 10 (2012).

[3] A. Mahadevan, V.L. Patel, N. Dagoglu, Oncology (Williston Park) 29, 743 (2015).

[4] M. Fort, S. Guet, S. Husheng, E. Calitchi, Y. Belkacemi, Crit. Rev. Oncol./Hematol. 99, 362 (2016).

[5] M. Banzi, S. De Blasio, A. Lallas, C. Longo, E. Moscarella, R. Alfano, G. Argenziano, Onco Targets Ther. 9, 2725 (2016).

[6] S. Martin-Algarra, R. Hinshelwood, S. Mesnage, et al., Melanoma Res. 29, 527 (2019).

[7] T. Gelb, S. Pshenichkin, O.C. Rodriguez, H.A. Hathaway, E. Grajkowska, J.O. DiRaddo, B. Wroblewska, R.P. Yasuda, C. Albanese, B.B. Wolfe, J.T. Wroblewski, Oncogene 34, 2711 (2015).

[8] T. Prickett, Y. Samuels, Clin. Cancer Res. 18, 4240 (2012).

[9] C.S. Williams, M. Mann, R.N. DuBois, Oncogene 18, 7908 (1999)

[10] K. Livak, T. Schmittgen, Methods 25, 402 (2001). 